\title{
Caracterización anatómica del leño y evolución del crecimiento en ejemplares de Acacia aroma y Acacia furcatispina en la Región Chaqueña, Argentina
}

\author{
Wood anatomical description and growth in individuals of Acacia aroma \\ and Acacia furcatispina in Chaco region, Argentina
}

\author{
Sandra Bravo ${ }^{1 *}$, Ana Giménez² , Juana Moglia ${ }^{2}$ \\ *Autor de correspondencia: ${ }^{1}$ Universidad Nacional de Santiago del Estero, Facultad de Ciencias Forestales, \\ Cátedra de Botánica, Av. Belgrano 11912 (s), Santiago del Estero, Argentina, sjbravo@correo.unse.edu.ar \\ ${ }^{2}$ Universidad Nacional de Santiago, Facultad de Ciencias Forestales, Cátedra de Dendrología.
}

\begin{abstract}
SUMMARY
The wood of Acacia aroma and Acacia furcatispina is employed for uses of little value such us posts, tools and firewood. The objectives of this work were to study anatomical characters of wood and to analyze the growth of isolated individuals with the purpose of determining their potential for uses of greater value. We determined that these species have high density and heavy wood, with diffuse porosity and tendency to semicircular. The wood of Acacia furcatispina has greater percentage of ligneous elements, better aesthetics qualities and health state than A. aroma. The most frequent defects in these species are rots within heartwood due to fungi attack, and discolorated wood formation, both related to fire scars. The mean width of the growth rings was $2.1 \mathrm{~mm}(\mathrm{DE}=0.47 \mathrm{~mm})$ in A. aroma and $1.9 \mathrm{~mm}(\mathrm{DE}=0.74 \mathrm{~mm})$ in A. furcatispina. In A. aroma, the curve of mean annual increment (IMA) intercepted the curve of current annual increment (ICA) for diameter at an age of 24 years, while in A. furcatispina it happened at 10 years. The results show that A. furcatispina is an interesting species to manage in plantations due to its wood qualities, although it would only allow small sized timber. This wood could be allocated for others uses such us parquets and artistic sculpture. Acacia aroma is a species suitable to manage for firewood production due to its capacity to produce greater diameter boles and branches, and to frequent wood decay that reduces its use for others more valuables uses.
\end{abstract}

Key words: wood of shrubs, growth of shrubs, wood anatomy.

RESUMEN

La madera de Acacia aroma y Acacia furcatispina se emplea para usos de escaso valor como postes, mangos de herramientas o combustible. Los objetivos de este trabajo fueron estudiar los caracteres anatómicos del leño de ambas especies y analizar el crecimiento de ejemplares aislados con la finalidad de determinar su potencialidad para usos de mayor valor. Se determinó que la madera de estas especies es dura, pesada y de porosidad difusa con tendencia a semicircular. Acacia furcatispina posee un leño con mayor porcentaje de elementos lignificados, mejores cualidades estéticas y mejor estado fitosanitario que el de A. aroma. Los defectos más frecuentes en estas especies fueron la formación de leño decolorado y pudriciones en la zona del duramen por ataque de hongos, asociados a la presencia de cicatrices de fuego. El espesor promedio de los anillos de crecimiento fue 2,1 mm $(\mathrm{DE}=0,47 \mathrm{~mm})$ en $A$. aroma y $1,9 \mathrm{~mm},(\mathrm{DE}=0,74 \mathrm{~mm})$ en A. furcatispina. Las curvas de incremento medio anual e incremento corriente anual del diámetro en A. aroma se interceptan a los 24 años mientras que las de A. furcatispina lo hacen a la edad de 10 años. Los resultados indicaron que A. furcatispina es una especie interesante para manejar en plantaciones debido a las cualidades de su madera, aunque permitiría obtener escuadrías menores. Su madera podría destinarse a usos particulares como parquets, tallas artísticas, entre otros. Acacia aroma es una especie apropiada para manejar con el objetivo de producción de leña debido a la capacidad de producir fustes y ramas de mayor diámetro y a que su estado fitosanitario limita su empleo para otros usos de mayor valor.

Palabras clave: leño de arbusto, crecimiento de arbustos, anatomía de leño.

\section{INTRODUCCIÓN}

Los bosques y arbustales de regiones áridas y semiáridas cumplen una función importante en relación a la protección del suelo contra la desertificación y representan una fuente de alimento para el hombre y los anima- les. A pesar de ello, las especies arbustivas suelen ser poco valoradas y se las emplea casi exclusivamente como combustible. Las especies de Acacia forman arbustales de considerable desarrollo en regiones tropicales y subtropicales de América, Australia y el sudeste de Asia. Sus especies tienen la capacidad de fijar nitrógeno 
atmosférico, proveen madera para combustibles, extractos medicinales, taninos, gomas, madera de obra, fibras, sombra y alimento para animales silvestres y domésticos (Casiva et al. 2002).

Dentro de la Región Chaqueña argentina las especies de Acacia pueden desarrollar porte arbóreo o arbustivo, según la edad y la calidad de sitio (Tortorelli 1956). Suelen formar bosquecillos secundarios puros denominados según la especie dominante, como tuscales, de Acacia aroma Gill. Ap. Hook. et Arn. o arcales de Acacia visco Lorentz ex Griseb. (Hueck 1978). A partir de las últimas décadas se han desarrollado distintas líneas de investigación en torno al manejo productivo de especies de Acacia con fines de uso múltiple (Thomson y Cole 1987, Lee et al. 1988, Sim y Gan 1991, Casiva et al. 2002, Plencovich et al. 2005). Algunos trabajos recomiendan para la forestación a A. furcatispina Burkart, A. caven (Mol.) Mol, A. atramentaria Benth. y A. aroma (Méndez 1993, Casiva et al. 2002) y en otros más recientes se analizan la productividad aérea y económica de arbustales de algunas especies del género (Plencovich et al. 2005).

El conocimiento de los caracteres anatómicos del leño y la determinación de tasas de crecimiento de especies arbustivas son necesarios para comprender mejor la dinámica de los arbustales, revalorizar su presencia e impulsar usos alternativos. Los objetivos de este trabajo fueron describir las características anatómicas del leño de A. aroma y A. furcatispina y analizar la evolución del crecimiento de árboles individuales con relación a la edad.

\section{MÉTODOS}

El área de estudio se localizó en el campo experimental "La María" perteneciente al Instituto Nacional de Tecnología Agropecuaria Santiago del Estero, INTA (28 $\left.3^{\prime} \mathrm{S}, 64^{\circ} 15^{\prime} \mathrm{O}\right)$. La misma se encuentra ubicada en la Región Chaqueña Occidental o Semiárida de Argentina, caracterizada por un clima semiárido y estacional, con el mayor déficit hídrico durante el verano (Boletta 1988). El paisaje chaqueño comprende un mosaico de bosques, sabanas, arbustales y pastizales de variada forma y tamaño (Brassiolo et al. 1993, López de Casenave et al. 1995). El muestreo de los ejemplares de A. aroma y A. furcatispina se hizo al azar en el ecotono de una sabana de Elionorus muticus Spreng. y bosques en distinto grado de desarrollo. La sabana corresponde al parche más grande de este tipo de vegetación dentro del campo experimental "La María", donde las especies en estudio se encuentran bien representadas y pueden alcanzar porte arbóreo o típicamente arbustivo, con ramas de orden semejante que nacen cerca del suelo (López de Casenave $e t$ al. 1995, Tálamo y Caziani 2003). Debido a la dureza de las maderas no se empleó barreno para la toma de muestras. Se apearon ocho ejemplares de cada especie, con buena vitalidad, incluyéndose individuos de porte arbóreo y arbustivo.

Se tomaron secciones transversales de leño sobre fuste o ramas principales, cada $0,30 \mathrm{~m}$ desde la base del ejemplar. Las muestras se cepillaron y lijaron hasta obtener superficies lisas con una buena demarcación de los anillos de crecimiento. La identificación de los anillos de crecimiento de A. aroma se hizo siguiendo las descripciones de Giménez y Moglia (1994). Se describieron para este trabajo los anillos de crecimiento de A. furcatispina. La marcación y medición del espesor de anillos de crecimiento se realizó con el equipo computarizado Aniol y el programa CATRAS (Aniol 1991). El espesor de anillos se determinó con una precisión de centésima de milímetro. Para realizar los ensayos sobre densidad se extrajeron dos probetas de $2 \mathrm{~cm}$ de arista de las secciones transversales de fuste de $0,30 \mathrm{~m}$ de altura, una correspondiente a la albura y la otra al duramen, a una distancia constante desde la médula. Para realizar los preparados microscópicos se obtuvieron probetas de las secciones correspondientes a $0,30 \mathrm{~m}$ de altura y se siguieron las normas tradicionales para el estudio de la madera (Baas et al. 1989). Las muestras se tiñeron con coloración triple de crisoidina-acridina roja y azul de astra, se deshidrataron en xilol y montaron en entellán (Freund 1970). En la descripción se empleó la terminología del Comité de Nomenclatura de IAWA (Baas et al. 1989). Se caracterizó el leño de las dos especies teniendo en cuenta histogramas de tejidos (porcentaje de vasos, de fibras, de parénquima axial y parénquima radial) y diámetro tangencial de vasos (Radermacher et al. 1984, Gourlay y Grime 1994).

Se analizaron las diferencias de medias de porcentajes de vasos, fibras, parénquima y densidad del xilema de A. aroma y A. furcatispina mediante la prueba de $t$ para muestras independientes, previo análisis de distribución de los datos y homogeneidad de las varianzas. Con los datos de espesores de anillos de crecimiento se construyó la curva promedio de diámetro de rodajas basales $(0,30$ $\mathrm{m}$ de altura) y se calculó el incremento medio anual (IMA) y el incremento corriente anual (ICA) para cada especie, dentro del rango de edades en la cual se dispone de datos para el $75 \%$ de los ejemplares de cada especie.

\section{RESULTADOS}

Características del leño. Acacia aroma tiene un leño con porosidad difusa con tendencia a semicircular (figura 1A). Los anillos de crecimiento están delimitados macroscópicamente por una línea clara que corresponde a un parénquima marginal formado por dos a tres estratos de células (figura 1B). Los miembros de vaso presentan un diámetro tangencial medio de 101,5 $\mu \mathrm{m}(57-132,5)$ y punteaduras escalariformes. El parénquima axial es del tipo paratraqueal vasicéntrico confluente en bandas irre- 
gulares con presencia de abundantes cristales en serie (figura 1C). El tejido fibroso alterna con las bandas de parénquima y presenta idioblastos con cristales rómbicos y hexagonales. Los radios leñosos son homogéneos, uni, bi o triseriados. En el duramen los vasos se presentan ocluidos por gomas. Se observa gomosis en parénquima y vasos, asociada a cicatrices de fuego.

La madera de $A$. aroma tiene una densidad básica promedio de $0,89 \mathrm{~kg} / \mathrm{dm}^{3}\left(\mathrm{DE}=0,08 \mathrm{~kg} / \mathrm{dm}^{3} \mathrm{n}=16\right)$. La densidad del duramen de esta especie es $0,93 \mathrm{~kg} / \mathrm{dm}^{3}$ $\left(\mathrm{DE}=0,08 \mathrm{~kg} / \mathrm{dm}^{3} \mathrm{n}=8\right)$ y la densidad de la albura es $0,86 \mathrm{~kg} / \mathrm{dm}^{3}\left(\mathrm{DE}=0,07 \mathrm{~kg} / \mathrm{dm}^{3} \mathrm{n}=8\right)$, siendo esta diferencia significativa $(P<0,05)$. La madera es de color castaño rojizo con veteado poco notable y el principal defecto es la pudrición de color blanco cremoso, asociada a la presencia de cicatrices de fuego. Estas lesiones producen tallos excéntricos con costillas, debido a la interrupción del crecimiento. En heridas de mayor magnitud se observa decoloración del leño y ataque de insectos, siendo las secciones más afectadas las correspondientes a 0,30 y $0,60 \mathrm{~m}$ (figura 2).

Acacia furcatispina tiene un leño con porosidad difusa con tendencia a semicircular (figura 3A). La demarcación de los anillos de crecimiento está dada por una banda de parénquima marginal formada por 5-8 estratos de células (figura 3B). Los miembros de vaso presentan un diámetro tangencial promedio de $94,1 \mu \mathrm{m}(57-137 \mu \mathrm{m})$ y punteaduras escalariformes. El parénquima es paratraqueal confluente en bandas irregulares y el tejido fibroso se dispone en estratos irregulares que alternan con bandas de parénquima. Se observa gomosis generalizada en anillos de crecimiento con cicatrices de fuego.
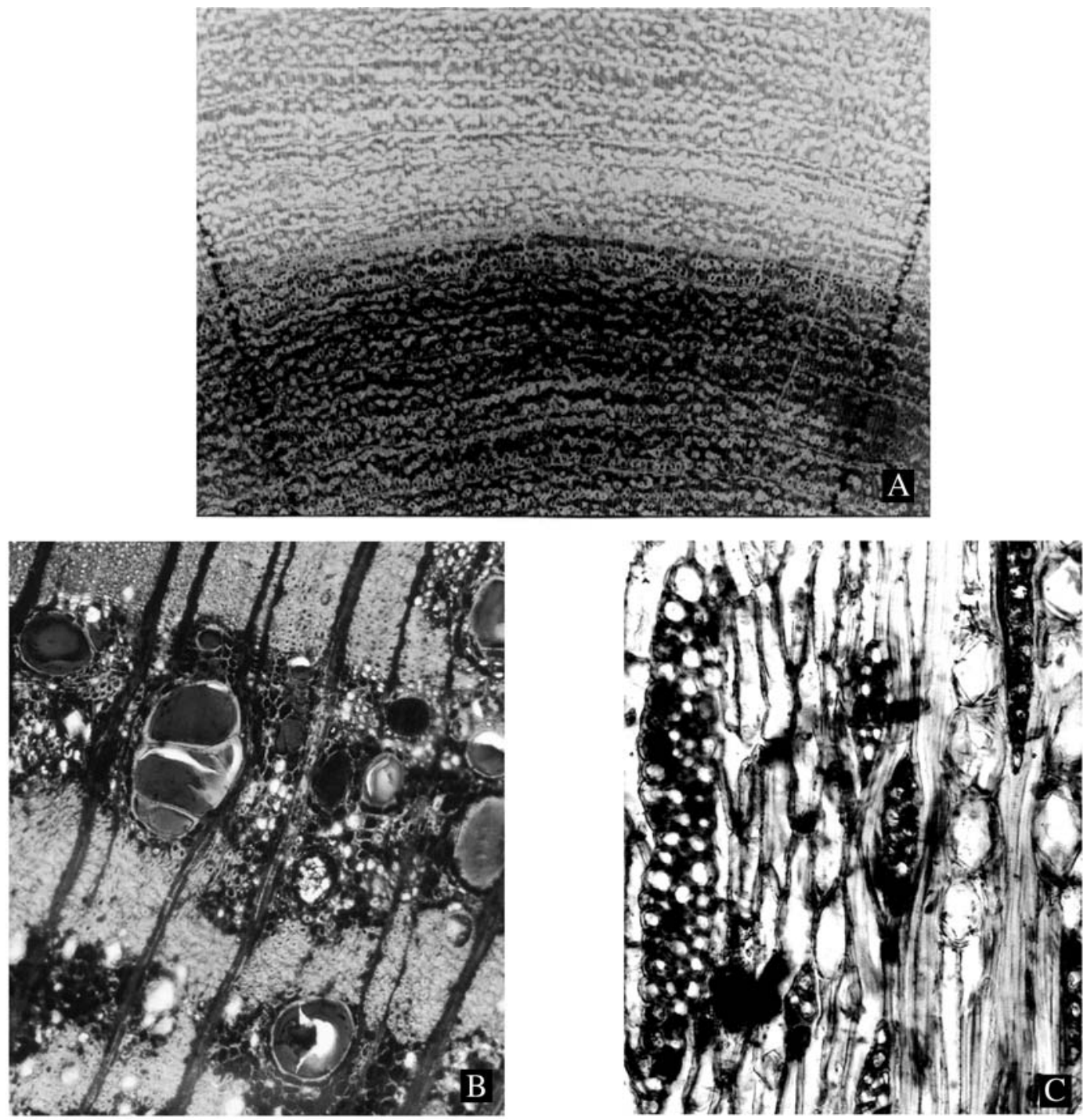

Figura 1. Leño de Acacia aroma A: macroscopía. B: detalle del leño en sección transversal (x25). C: cristales en parénquima axial, sección longitudinal tangencial (x40).

Wood of Acacia aroma. A: Macroscopic transversal section of wood. B: Microscopic transversal section of wood (x25). C: Cristals in axial parenchyma, tangential longitudinal section $(\mathrm{x} 40)$. 

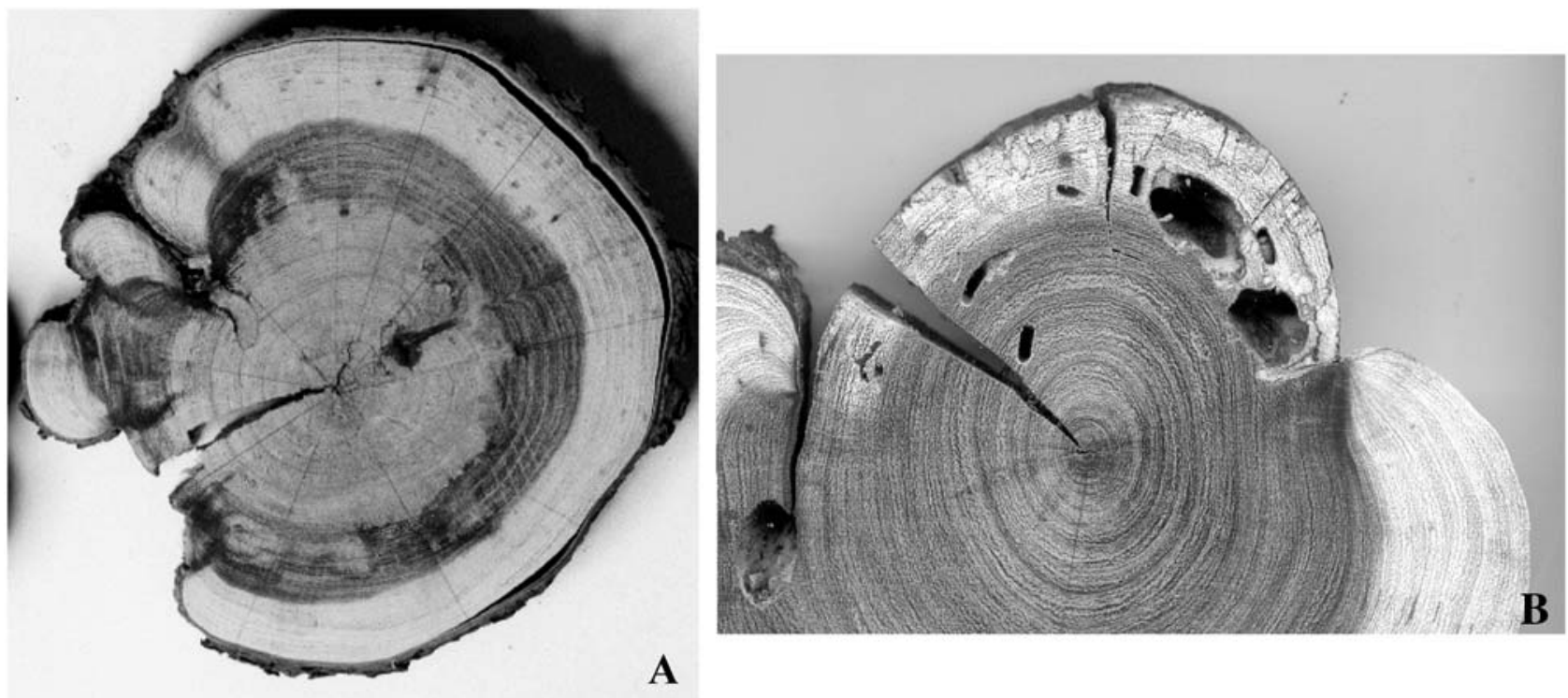

A

Figura 2. Defectos del leño de Acacia aroma. A: pudrición blanca. B: leño decolorado relacionado a cicatriz de fuego y daños por galerías de insectos.

Defects in Acacia aroma. A: White rot and ribs. B: discolored wood related to fire scars, damages caused by insects.
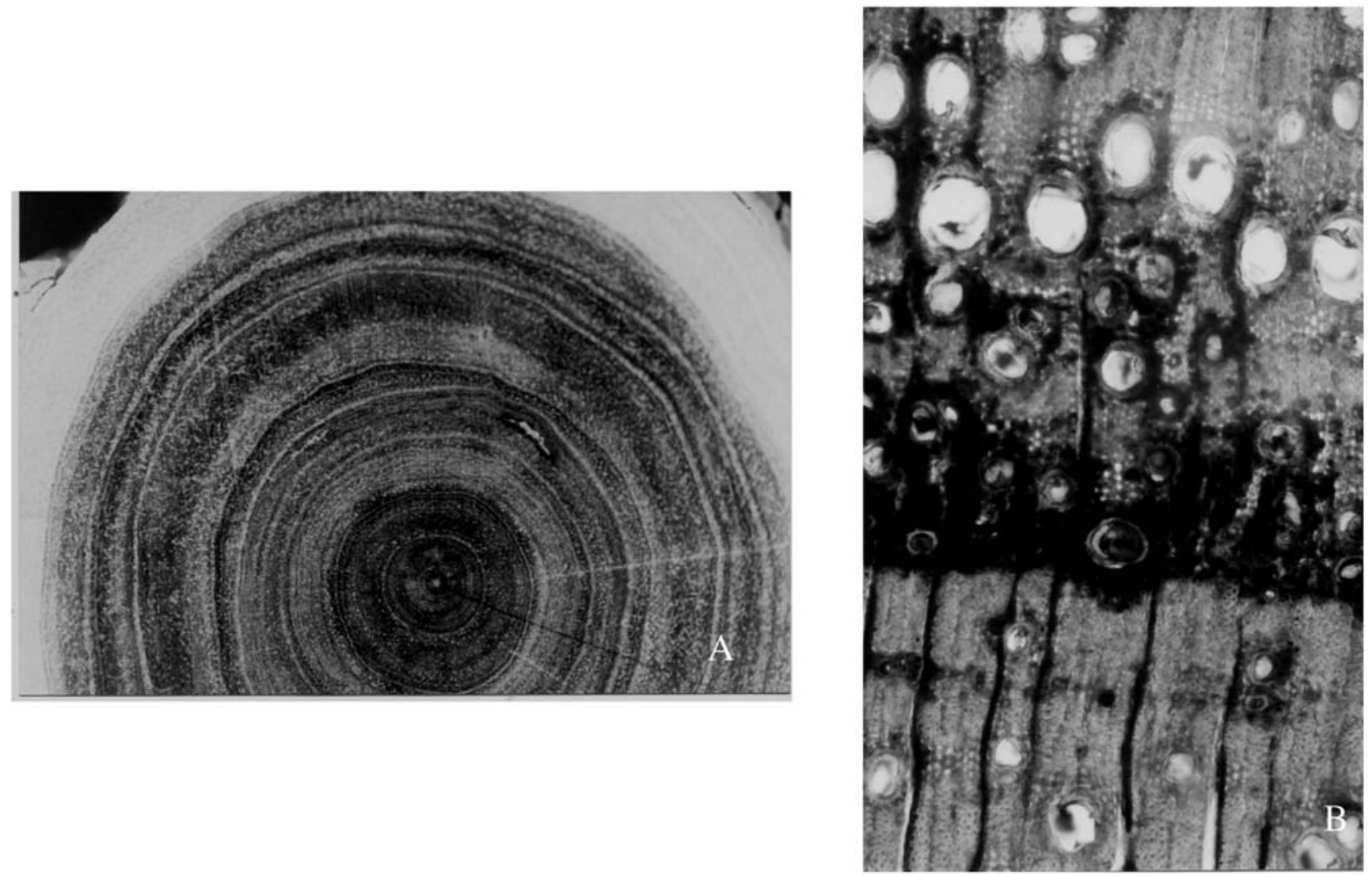

Figura 3. Leño de Acacia furcatispina. A: macroscopía. B: microscopía del leño en sección transversal (x25).

Wood of A. furcatispina. A: Macroscopic transversal section of wood. B: Microscopic transversal section of wood (x25). 

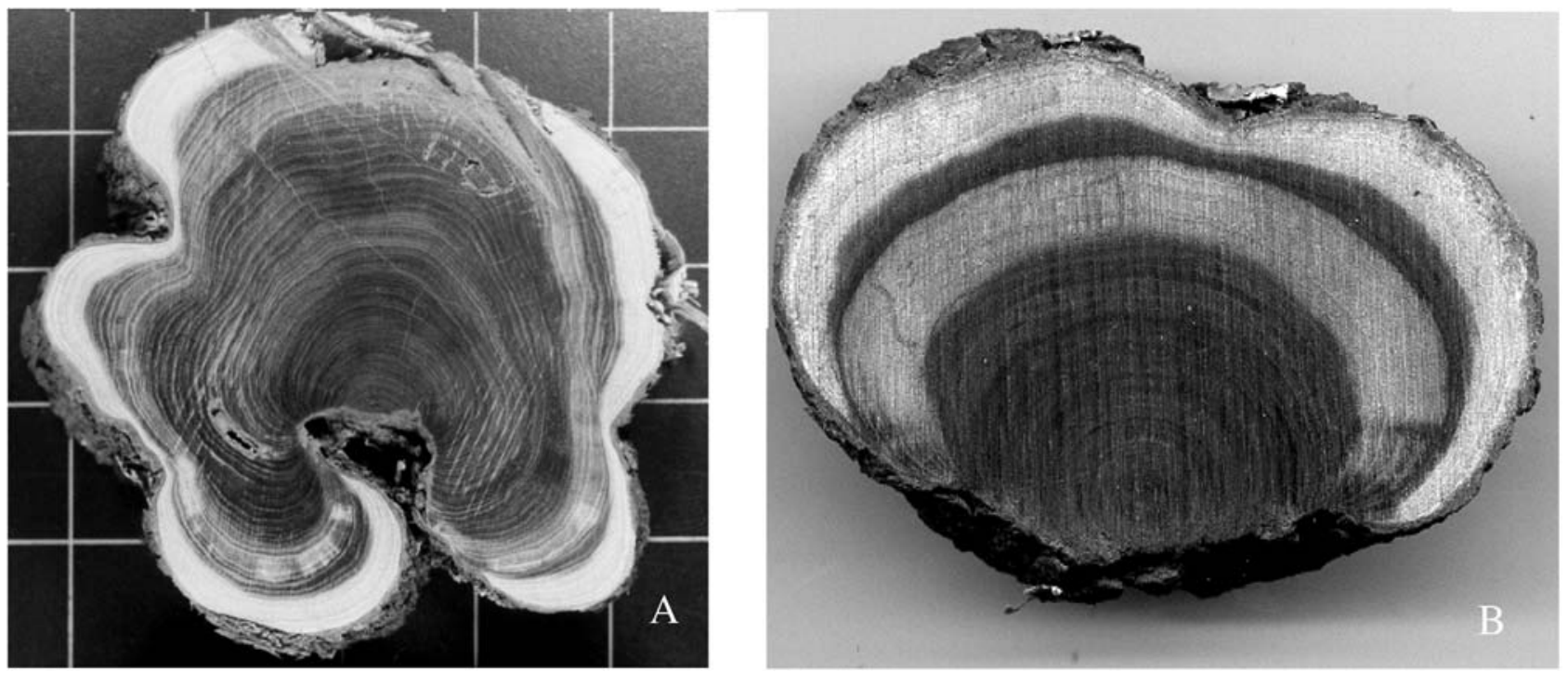

Figura 4. Defectos del leño en Acacia furcatispina relacionado a cicatrices de fuego. A: formación de costillas. B: decoloración del duramen.

Defects in in Acacia furcatispina wood related to fire scars. A: Ribs. B: Ring of discolored wood.

La madera de A. furcatispina tiene una densidad básica media de $0,99 \mathrm{~kg} / \mathrm{dm}^{3}\left(\mathrm{DE}=0,07 \mathrm{~kg} / \mathrm{dm}^{3}, \mathrm{n}=14\right)$. La densidad del duramen de esta especie es $1,01 \mathrm{~kg} / \mathrm{dm}^{3}$ $\left(\mathrm{DE}=0,07 \mathrm{~kg} / \mathrm{dm}^{3} \mathrm{n}=7\right)$ y la densidad de la albura es $0,97 \mathrm{~kg} / \mathrm{dm}^{3}\left(\mathrm{DE}=0,07 \mathrm{~kg} / \mathrm{dm}^{3} \mathrm{n}=7\right)$, siendo esta diferencia significativa $(P<0,05)$. La madera es de color castaño violáceo y el parénquima marginal que define los anillos de crecimiento produce un veteado notable en líneas verticales. El principal defecto es la presencia de leño decolorado y la formación de costillas, relacionadas ambas a cicatrices de fuego (figura 4). Las columnas de leño decolorado pueden extenderse más de $2 \mathrm{~m}$ de altura en sentido longitudinal. En sólo dos ejemplares de esta especie se han detectado pudriciones blancas de corazón.

El leño de A. furcatispina tiene mayor porcentaje de vasos y fibras y menor porcentaje de parénquima axial y radial que el leño A. aroma, siendo tales diferencias significativas $(P<0,05)$ sólo para el primero y el último de los caracteres mencionados (cuadro 1). Esto se relaciona con la mayor densidad del leño de A. furcatispina $\left(t_{13}=-3,51, P=0,001\right)$.

Espesor de anillos de crecimiento. El espesor promedio de los anillos de crecimiento de A. aroma es de $2,1 \mathrm{~mm}$ $(0,58-6,31 \mathrm{~mm}, \mathrm{DE}=0,47 \mathrm{~mm})$, según lo determinado en ejemplares cuyas edades oscilaron entre 20 a 41 años. El espesor medio de los anillos de crecimiento en función de la edad se ajusta a una ecuación polinómica de tercer grado con $\mathrm{R}^{2}=0,5095$ (hasta la edad de 31 años) y alcanza un valor máximo de $2,5 \mathrm{~mm}$ a los 14 años (figura 5A). El espesor promedio de los anillos de creci-
Cuadro 1. Composición porcentual de los distintos elementos del leño de Acacia aroma y Acacia furcatispina. Letras diferentes indican diferencias significativas entre especies $(P<0,05)$.

Percentage composition of different elements of Acacia aroma and Acacia furcatispina's wood. Different letters indicate significant differences $(P<0.05)$.

\begin{tabular}{ccccc}
\hline \multirow{2}{*}{$\begin{array}{c}\text { Componente } \\
\text { del leño }\end{array}$} & \multicolumn{3}{c}{ Acacia aroma } & \multicolumn{3}{c}{ Acacia furcatispina } \\
Media & $\mathrm{S}$ & Media & $\mathrm{S}$ \\
\hline Vasos & $10,4 \mathrm{a}$ & 2,5 & $21,8 \mathrm{~b}$ & 4,6 \\
Fibras & 36,2 & 3,6 & 42,0 & 8,9 \\
Parénquima axial & 32,2 & 5,8 & 28,4 & 9,3 \\
Parénquima radial & $21,2 \mathrm{a}$ & 6,4 & $7,8 \mathrm{~b}$ & 3,0 \\
\hline
\end{tabular}

S: desviación estándar.

miento en A. furcatispina es $1,9 \mathrm{~mm}(0,67-4,69 \mathrm{~mm}$, $\mathrm{DE}=0,74 \mathrm{~mm}$ ), según lo determinado en ejemplares cuyas edades oscilaron entre 22 a 57 años. El espesor medio de los anillos de crecimiento y la edad se ajusta a una ecuación polinómica de tercer grado con $\mathrm{R}^{2}=0,7747$ (hasta una edad de 27 años) y presenta un valor máximo de 2,3 $\mathrm{mm}$ a los 12 años. Estos son los primeros datos en relación a espesores de anillos de crecimiento para esta especie (figura 5B).

Evolución del diámetro con la edad. La curva promedio de evolución del diámetro de las rodajas basales con la edad ajusta en A. aroma a una ecuación polinómica de tercer orden con $\mathrm{R}^{2}=0,999$, hasta la edad de 31 años a la que alcanzan el $75 \%$ de los ejemplares estudiados 

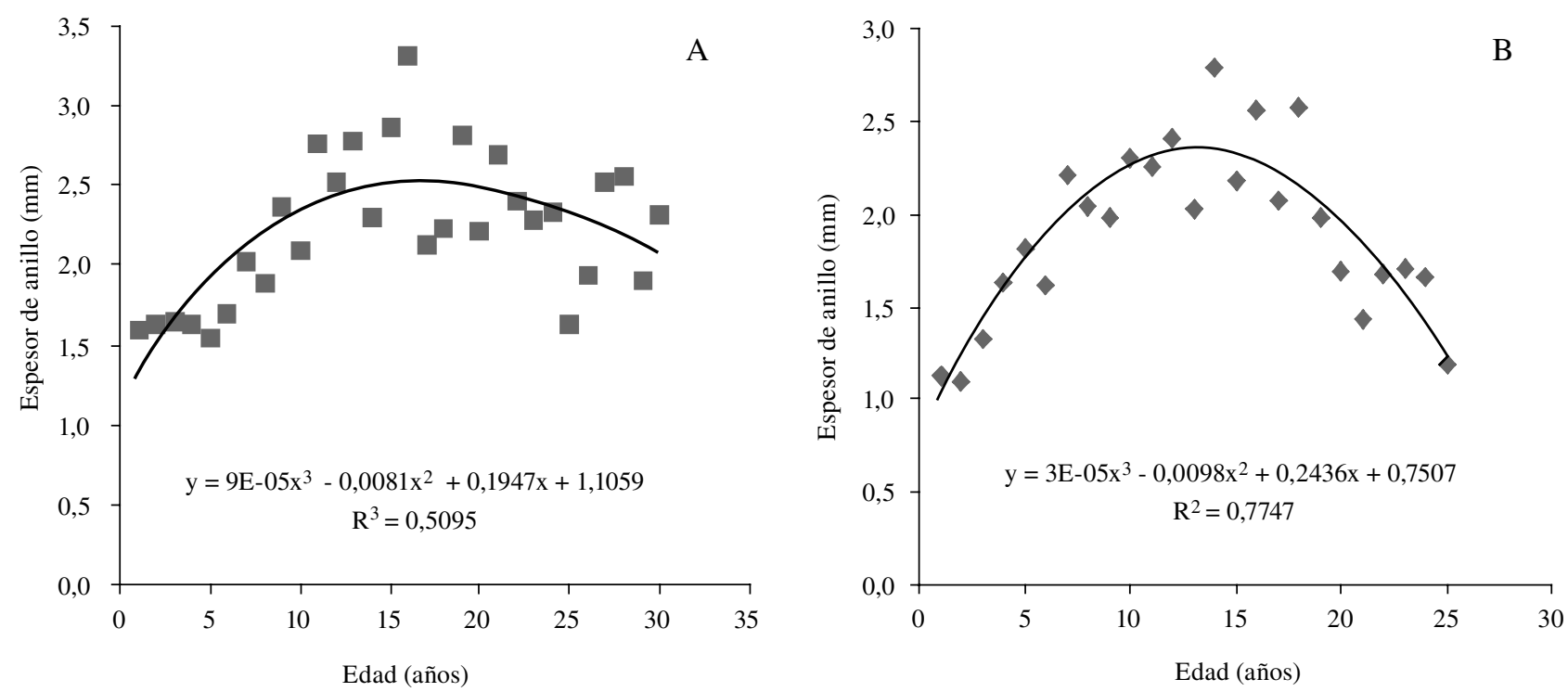

Figura 5. Espesor de anillos de crecimiento y edad. A) A. aroma. B) A. furcatispina.

Figure 5. Width of the growth rings and age. A: Acacia aroma. B: Acacia furcatispina.
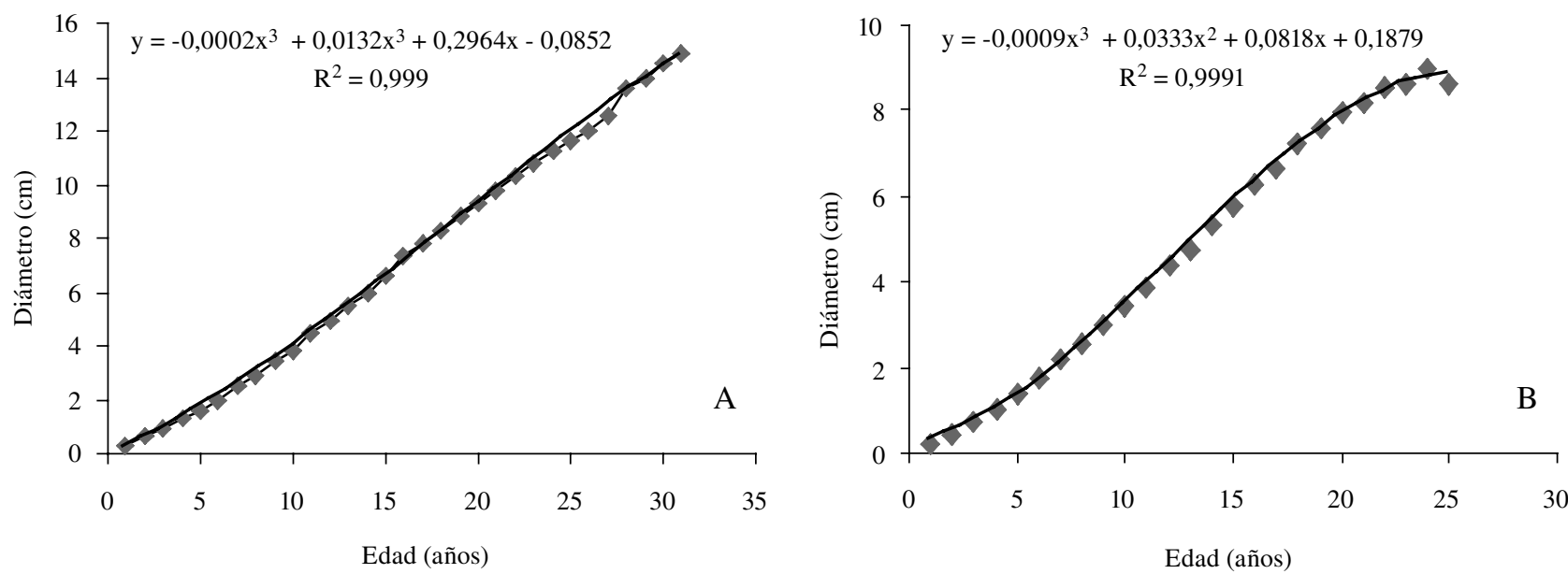

Figura 6. Evolución del diámetro con la edad. A) Acacia aroma ( $\mathrm{n}=8$ ejemplares). B) Acacia furcatispina ( $\mathrm{n}=8$ ejemplares). Diameter evolution with age. A: Acacia aroma ( $\mathrm{n}=8$ individuals). B: Acacia furcatispina ( $\mathrm{n}=8$ individuals).

(figura 6A). En A. furcatispina esta curva ajusta a una ecuación polinómica de tercer orden con $\mathrm{R}^{2}=0,999$, hasta la edad de 25 años a la que alcanza el $75 \%$ de los ejemplares estudiados (figura 6B). En esta especie el ejemplar de mayor edad alcanza un diámetro de $16 \mathrm{~cm}$ a los 57 años.

En A. aroma se observa un IMA máximo $0,45 \mathrm{~cm}$ de diámetro a los 22 años y en A. furcatispina es de $0,41 \mathrm{~cm}$ de diámetro a las edades comprendidas entre 9 y 10 años (figura 7A). Las curvas de IMA e ICA del diámetro se interceptan en A. aroma a la edad de 24 años, mientras que en A. furcatispina lo hacen a la edad de 10 años (figura 7B). En la figura 8 se representan el IMA e ICA del diámetro de las secciones basales de los ejemplares más añosos de cada una de las especies.

\section{DISCUSIÓN}

La porosidad y la delimitación de anillos de crecimiento de A. aroma y A. furcatispina concuerdan con lo descrito por Gourlay y Grime (1994) como característico de las especies del género Acacia. Según Giménez (1993) la porosidad difusa con tendencia a semicircular de $A$. aroma se encuentra relacionada a la notable estacionalidad de la actividad cambial impuesta por el régimen hídrico de la Región Chaqueña semiárida. La demarcación de los anillos de crecimiento es más notable en $A$. furcatispina debido al mayor desarrollo del parénquima marginal, lo que, a su vez, produce en la madera un veteado en líneas verticales muy vistoso. La gomosis relacionada a las cicatrices del fuego representa una respuesta común 

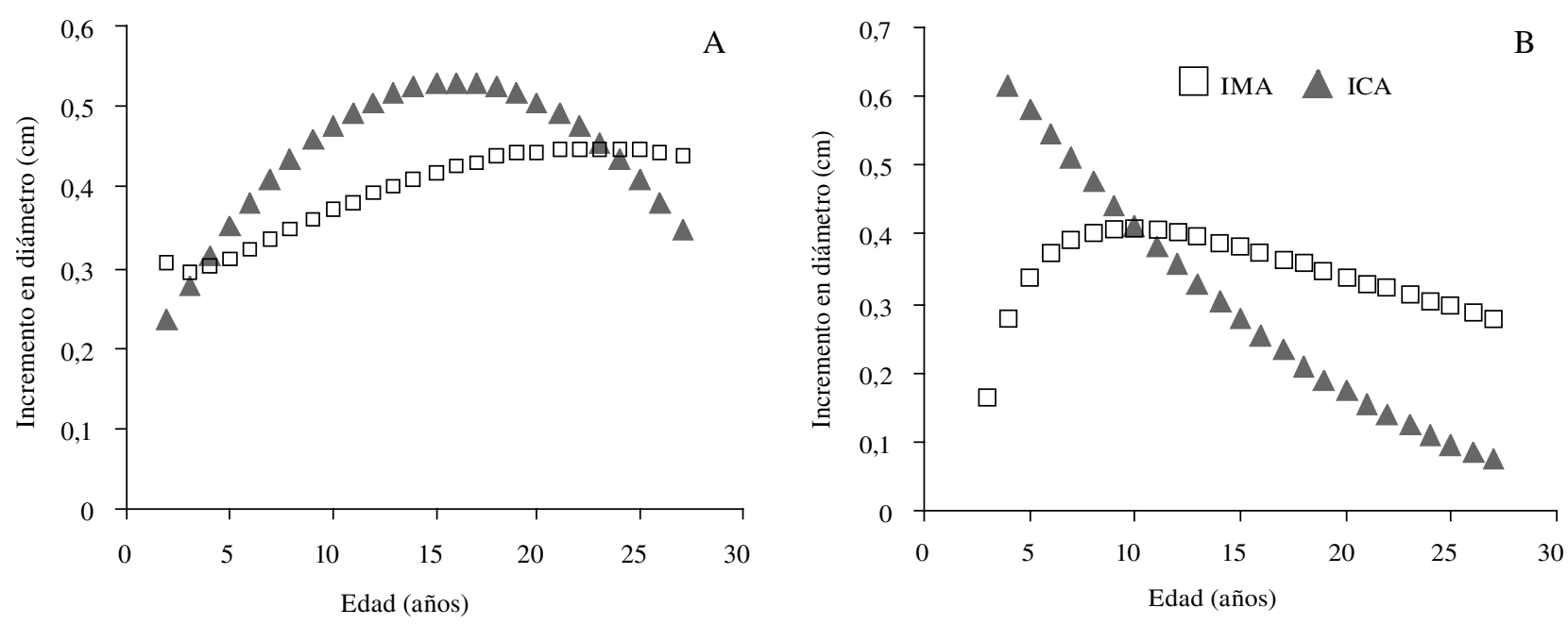

Figura 7. Incremento medio anual (IMA) e incremento corriente anual (ICA) de diámetro de fuste A) Acacia aroma, B) Acacia furcatispina.

Mean annual increment (IMA) and current annual increment (ICA) of diameter of bole.
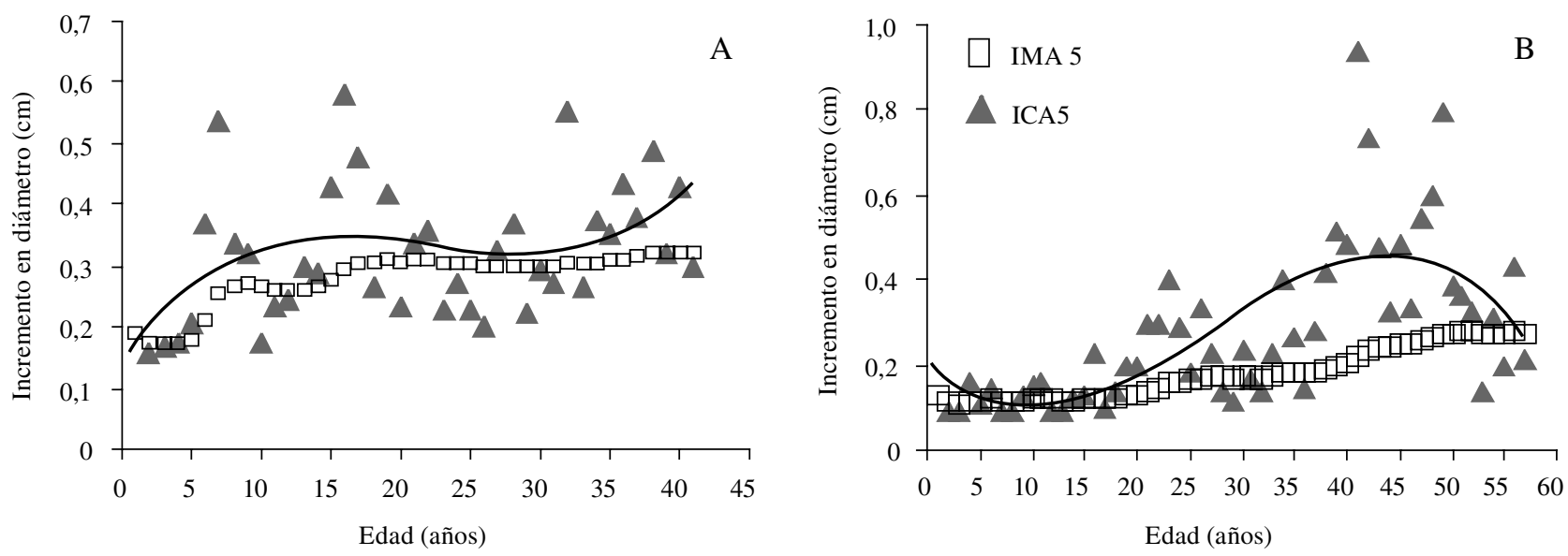

Figura 8. Incremento medio anual (IMA) e incremento corriente anual (ICA) en diámetro de fuste, en los ejemplares de mayor edad A) Acacia aroma, B) Acacia furcatispina.

Mean annual increment (IMA) and current annual increment (ICA) of diameter of bole in older individuals.

frente a daños al tejido cambial (Larson 1994, Giménez et al. 1998, Bravo et al. 2001). Así también la presencia de abundantes cristales en el leño de A. aroma es considerado un carácter frecuente entre las especies de zonas áridas (Gourlay y Grime 1994).

El diámetro tangencial medio de los vasos permite asignar la categoría de "poros medianos" a A. aroma y "poros pequeños" a A. furcatispina, según las categorías establecidas por Giménez (1993). Este resultado concuerda con el mayor porcentaje de vasos en la madera de A. furcatispina, ya que existe una relación inversa entre el número de poros y el diámetro tangencial de sus elementos (Moglia y López 2001). El mayor porcentaje de elementos lignificados influye probablemente en el mejor estado fitosanitario observado en las muestras de leño de A. furcatispina. En A. aroma, el parénquima axial y radial brindan probablemente una mayor cantidad de sustrato respirable para patógenos como bacterias y hongos, responsables de pudriciones de corazón en muestras de leño. Este tipo de deterioro ha sido observado en el leño de otras especies de Acacia, citándose como hongos patógenos a especies de los géneros Fomes y Ganoderma (Lee et al. 1988).

La decoloración del leño observada en A. furcatispi$n a$ es un defecto que disminuye la calidad de la madera y representa, al igual que la gomosis y otros procesos de secreción, una respuesta común frente a daños al tallo (Radermacher et al. 1984, Giménez et al. 1998, Bravo et al. 2001, Smith y Sutherland 2001). En A. mangium la decoloración del leño es inducida por la invasión de 
patógenos a través de una lesión (Lee et al. 1988, Schmitt et al. 1995). Bravo et al. (2001) determinaron en Prosopis alba Griseb. y Prosopis nigra (Griseb.) Hieron que las cicatrices y marcas de fuego inducen la formación de leño decolorado.

La densidad del leño de A. furcatispina y A. aroma se encuentra dentro del rango considerado correspondiente a maderas duras y pesadas, lo que amplía el espectro de usos, ya que indica la posibilidad de obtener piezas de muy buen acabado (Tortorelli 1956, Giménez y Moglia 2003). La mayor densidad promedio de la madera de A. furcatispina se encuentra vinculada al mayor porcentaje de vasos y fibras y a la menor proporción de parénquima radial. La diferencia significativa entre la densidad de albura y duramen en ambas especies puede relacionarse con el proceso de duraminización del leño, caracterizado por el depósito de abundantes contenidos celulares (Giménez y Moglia 2003).

El espesor promedio de los anillos de crecimiento de $A$. aroma de $2,1 \mathrm{~mm}$ se encuentra dentro del rango de espesor de anillos de crecimiento determinado para esta especie por Giménez y Moglia (1993). El mayor espesor de anillos de crecimiento le permite a $A$. aroma alcanzar diámetros de aproximadamente $20 \mathrm{~cm}$ a edades cercanas a los 30 años, mientras que en A. furcatispina sólo el más viejo de los ejemplares alcanza diámetros de $16 \mathrm{~cm}$ a la edad de 57 años (figura 6). Méndez (1993) comunica diámetros de hasta $30 \mathrm{~cm}$ en ejemplares de A. furcatispina creciendo en áreas riparias de la región fitogeográfica del monte, lo que podría indicar que la especie posee la potencialidad de desarrollar fustes de mayor diámetro en áreas donde el aporte hídrico no sea marcadamente limitante.

El punto de intercepción de las curvas correspondientes al IMA e ICA del diámetro indica que la culminación del crecimiento en diámetro de A. aroma ocurre a edades más avanzadas que en $A$. furcatispina. Las curvas de IMA e ICA de diámetros de rodajas basales de los ejemplares de mayor edad, de cada una de las especies, sugiere que la edad de culminación de crecimiento puede prolongarse. Esto puede atribuirse a la variabilidad propia de los ejemplares de una misma población (Villagra et al. 2002). Cabe resaltar que son estos los primeros datos en torno al crecimiento de estas especies arbustivas chaqueñas y son necesarios mayores estudios para establecer pautas apropiadas de manejo.

\section{CONCLUSIONES}

Acacia aroma y A. furcatispina poseen leños con características consideradas deseables para especies destinadas a aprovechamiento forestal. La densidad de sus leños se encuentra dentro de los rangos de maderas duras y pesadas. Los datos generados en relación a espesor de anillos de crecimiento y crecimiento en diámetro sugie- ren que $A$. aroma es apta para producción de leña, ya que la culminación de crecimiento ocurre a edades más avanzadas. La densidad, las características de la madera y el estado fitosanitario de A. furcatispina la convierten en una especie interesante para manejar en plantaciones con prácticas como raleo, poda, riego y control de incendios, ya que permite obtener madera de muy buena calidad, aunque de escuadrías menores.

Estos son los primeros datos obtenidos en relación al crecimiento de arbustos chaqueños y confirman la necesidad de generar más información en torno a este tema. Esto permitirá optimizar la utilización de los recursos del bosque, más allá de las especies tradicionalmente consideradas valiosas para la obtención de madera y otros usos.

\section{REFERENCIAS}

Aniol R. 1991. Computer Aided Tree Rings Analysis System, User Manual. Schleswing, Alemania. 31 p.

Baas P, E Wheeler, P Gasson. 1989. IAWA List of Microscopy Features for Hardwoods Identification. IAWA Committee. 322 p.

Boletta P. 1988. Clima, desmonte y habilitación de tierras en la región chaqueña semiárida. Santiago, Chile. FAO, Casas $\mathrm{R}$ (editor). $306 \mathrm{p}$.

Brassiolo M, R Renolfi, W Grafe, E. Fumagalli. 1993. Manejo Silvo-pastoril en el Chaco Semiárido. Quebracho 1:15-28.

Bravo S, A Giménez, J. Moglia. 2001. Efectos del fuego en la madera de Prosopis alba Griseb. y Prosopis nigra (Griseb.) Hieron, Mimosaceae. Bosque 22(1):51-63.

Casiva P, B. Saidman, J Vilardi, A Cialdella. 2002. First comparative phenetic of argentinian species of Acacia (Fabaceae), using morphometric, isozymal and RAPD approaches. American Journal of Botany 89:843-853.

Deschamps J, J Wright. 1997. Patología forestal de Cono Sur de América. Buenos Aires, Argentina. Orientación Gráfica Editora. 237 p.

Freund H. 1970. Handbuch der Mikroscopie in der Technik. Frankfurt, Alemania. Umschan Verlag. 379 p.

Giménez A. 1993. Rasgos estructurales característicos del leño secundario de las especies arbóreas de la región chaqueña seca. Quebracho 1:1-14.

Giménez A, J Moglia. 1994. Determinación de patrones de crecimiento de especies leñosas arbóreas de la región Chaqueña Seca. Universidad Nacional de Misiones. Yvyraretá 4:46-60.

Giménez A, N Ríos, J Moglia, C. López. 1998. Leño y corteza de Prosopis alba Griseb. algarrobo blanco, Mimosaceae, en relación con algunas magnitudes dendrométricas. Bosque 19 (2):53-62.

Giménez A, J Moglia. 2003. Árboles del Chaco argentino. Guía para el reconocimiento dendrológico. Santiago del Estero, Argentina. Universidad Nacional de Santiago del Estero y Secretaría de Ambiente y Desarrollo Sustentable. 306 p.

Gourlay I, G Grime. 1994. Calcium oxalate crystals in african Acacia species and their analysis by scanning proton microprobe. IAWA Journal 15 (2):137-148. 
Hueck K. 1978. Los Bosques de Sudamérica. Sociedad Alemana de Cooperación Técnica. 476 p.

Larson P. 1994. The Vascular Cambium. Development and structure. Springer Series in Wood Science. 726 p.

Lee S, S Teng, M Lim, R Abd, Kader. 1988. Discoulouration and heart rot of Acacia mangium Willd. Some preliminary results. Journal of Tropical Forest Science 1 (2):170-177.

López de Casenave J, J Pelotto, J Protomastro. 1995. Edgeinterior differences in vegetation structure and composition in a Chaco semi-arid forest, Argentina. Forest Ecology and Management 72:61-69.

Méndez E. 1993. Bosques de Acacia furcatispina (garabato) en cerrilladas pedemontanas de Mendoza. Multequina 2:157-161.

Moglia J, C López 2001. Variabilidad radial de algunos caracteres anatómicos de Aspidosperma quebracho blanco. Bosque 22(2):3-14.

Perpiñal E, M Balzarini, L Catalán, L Pietrarelli, U Karlin. 1995. Edad de culminación de crecimiento en Prosopis flexuosa D.C. en el Chaco Árido argentino. INIA. Investigaciones Agrarias, Sistemas y Recursos Forestales 4 (1):45-55.

Plencovich C, R Coirini, R Zapata. 2005. Productividad aérea y económica de Acacia caven (Mol) Hook. en bosques nativos del espinal entrerriano. Actas Resúmenes del III Congreso Nacional sobre Manejo de Pastizales Naturales, Paraná, Entre Ríos, Argentina. 100 p.
Radermacher J, J Bauch, A Shigo. 1984. Characteristics of xylem formed after wounding in Acer, Betula and Fagus. Development and characteristics of discoloured woods. IAWA Bulletin 5(2):141-151.

Smith K, E Sutherland. 2001. Terminology and biology of fire scars in selected Central Hardwoods. Tree Ring Research 57:141-147.

Sim B, E Gan. 1991. Performance of Acacia species on four sites of Sabah forest industries. Advances in Tropical Acacia Research. Thailandia. Proceedings of an Internacional Workshops in Bangkok.

Tálamo A, S Caziani. 2003. Variation in woody vegetation among sites with different disturbance histories in the Argentine Chaco. Forest Ecology and Management 184:79-92.

Thomson L, E Cole. 1987. Colecciones de semillas de especies leñosas en Australia tropical, árida y semiárida y recomendaciones para pruebas internacionales de especies. Recursos Genéticos Forestales. Informe $\mathrm{N}^{\circ} 15$. ONU. Agricultura y Alimentación. p. 37-50.

Tortorelli L. 1956. Maderas y Bosques Argentinos. Buenos Aires, Argentina. Editorial ACME. 891 p.

Villagra P, M Morales, R Villalba, J Boninsegna. 2002. Dendroecología de los algarrobales de la zona árida argentina. Instituto Argentino de Nivología, Glaciología y Ciencias Ambientales IANIGLA 1973-2002. p. 53-57. 